\title{
Greenspace availability or greenspace usability, which matters on PM2.5-related premature deaths: evidence from 360 cities in China
}

Jingru Yang

Beijing Forestry University

Yijin Wang

Beijing Forestry University

Fangzheng Li ( $\sim$ fangzhengli@bjfu.edu.cn )

Beijing Forestry University

Yuge Xie

Beijing Forestry University

Xiaoli Wang

Beijing TsingHua TongHeng Urban Planning and Design Institute

Article

Keywords: Greenspace exposure, air pollution, usability

Posted Date: November 23rd, 2021

DOI: https://doi.org/10.21203/rs.3.rs-1074052/v1

License: (a) This work is licensed under a Creative Commons Attribution 4.0 International License. Read Full License 


\title{
Greenspace availability or greenspace usability, which matters on
}

\section{PM 2.5 -related premature deaths: evidence from 360 cities in China}

\begin{abstract}
Greenspace exposure is confirmed to reduce air pollution-related negative health impact. However, which type of greenspace exposure matters more on mitigating air pollution-related deaths and whether this effect is regionally different remain unclear. Here we show, greenspace usability exposure plays a more significant role in mitigating $\mathrm{PM}_{2.5}$-related premature deaths in 360 China cities generally speaking. By clustering 360 cities into urban-rural and Deprivation Index groups, we further find that greenspace availability and usability together work on respiratory health in rural regions, and greenspace availability matters more in very low deprived areas or urban and rural regions. Our results that increasing greenspace usability exposure is more helpful in reducing air pollution-related premature deaths may inform more effective and equitable greenspace planning policies in rapidly developing countries like China.
\end{abstract}

\section{Introduction}

Air pollution is the fifth highest leading cause for premature deaths globally ${ }^{1,2,3,4}$. Nearly $86 \%$ populations in expanding developing countries such as China, India, and Bangladesh experienced the extreme $\mathrm{PM}_{2.5}$ concentrations ${ }^{5,6}$. It shows that the problem of $\mathrm{PM}_{2.5}$-related premature deaths are becoming a global respiratory health challenge rather than a regional air pollution issue ${ }^{7,8}$. Many studies have proved the positive effect of greenspace on reducing $\mathrm{PM}_{2.5}$ exposure and promoting environment restoration $9,10,11,12,13,14$. Whereas no studies have quantified whether greenspace can mitigate $\mathrm{PM}_{2.5}$-related health impact by relieving $\mathrm{PM}_{2.5}$ emissions. In response to achieving high-quality development, the epidemiological evidence associating greenspace and respiratory health has put greenspace interventions as a sustainable way to reduce air pollutionrelated premature mortality on the agenda of planners and policymakers ${ }^{14,15}$.

Existing literature indicates that exposure to greenspace reduces the risk of all-cause premature mortality $8,9,12$, and broadly classify the greenspace exposure into two aspects; greenspace availability exposure and greenspace usability exposure ${ }^{1,16,17}$. Greenspace availability such as normalized difference vegetation index (NDVI) has widely proven to have unequal positive effect on residents' respiratory health in regions with different socioeconomic levels ${ }^{18,19}$. It still remains unclear that which type of greenspace exposure could matters more on alleviating air pollutionrelated premature deaths? Although most studies give an overall assessment of greenspace quantity for the entire study area, few studies can reveal residents' actual exposure to greenspace from a large scale ${ }^{20}$. Besides, differences of local natural base and green infrastructure construction have led to strong inequity of people's access to greenspace ${ }^{21,22}$. Simply considering the relationship between total amount of greenspace and air pollution-related health seems to have encountered bottlenecks $1,10,23,24,25$. Thus, working on combining human mobility and greenspace distribution will become an inevitable trend. Additionally, emissions, urban structure, and socioeconomic factors such as $\mathrm{PM}_{2.5}$ concentration, population density and GDP are also significantly linked with air pollutionrelated premature deaths, and their impact on $\mathrm{PM}_{2.5}$-related health effect need to be discussed together with greenspace exposure ${ }^{8,19,26,27}$. It's also remarkable that these factors and premature deaths have obvious regional heterogeneity because of differences in policy implementation and 
socioeconomics ${ }^{1,18}$. On account of the inconsistent progress of China's clean air plan in cities, strong emission control of high-density urban areas may thus encourage air pollution to transfer or leak to less developed areas ${ }^{2,7}$. Yet these areas have less resources to provide health services and construct green infrastructure, which will further aggravate the health threat caused by air pollution

7,28 . Whereas numerous studies have examined the difference in air pollution-related morbidity or deaths in urban and rural areas, or cities with different deprivation indexes ${ }^{1,18,28}$. No investigation has associated the positive effect of greenspace exposure on respiratory health with city structure and socioeconomic differences. Regarding the inequity of respiratory health in different affluent areas, whether greenspace quantity or greenspace experience or a combination of this two has a positive effect will be potential to affect decisions of policymakers on zone planning ${ }^{29,30}$.

In the present study, using multiple linear regression model, we quantify the relationship between greenspace exposure and $\mathrm{PM}_{2.5}$-related premature deaths in 360 cities in whole China. First, we identify the outdoor $\mathrm{PM}_{2.5}$-related premature deaths by using the integrated exposure-response (IER) concentration-response relationships ${ }^{7}$. Second, we use big datas including NDVI, LAI, LULC and per capita green space exposure to characterize the greenspace exposure. Then, we cluster group the samples based on Deprivation Index, and identify the differences in the relationship between greenspace exposure and $\mathrm{PM}_{2.5}$ related premature death in each group. Our work provides quantitative estimates of greenspace exposure and $\mathrm{PM}_{2.5}$ related premature deaths in large scale from a city planning-based perspective, and reveal the different role of greenspace availability and greenspace usability in urban, rural and further deprivation levels. The findings of this study provide implications on targeted and equitable greenspace planning for mitigation plans on public respiratory health in China.

\section{Result}

Descriptive data. Figure 1 presented the outdoor $\mathrm{PM}_{2.5}$-related premature deaths (person), greenspace availability exposure and greenspace usability exposure in 360 Chinese cities. A total of 2,841,180 $\mathrm{PM}_{2.5}$-related premature deaths were obtained in all 360 cities in china during the study period, and 503,905 $\mathrm{PM}_{2.5}$-related premature deaths from 17 megacities accounted for $17.74 \%$ of the total. The greenspace usability exposure ranged from 3.22E-07to $3.30 \mathrm{E}-03$ square meter per person and the greenspace availability exposure ranged from 0.22 to 0.68 . We observed that high greenspace usability exposure regions were mostly concentrated in woodland-rich cities while high greenspace availability exposure regions were mostly concentrated in cities with a medium economic level, sufficient green space and moderate population density (Fig.1, Fig. 4). High $\mathrm{PM}_{2.5}$ concentrations regions were also noticed that distributing in cities with developed industries or dense populations (Fig.4). Descriptive information on the number of $\mathrm{PM}_{2.5}$ related premature deaths and the greenspace exposure are presented in Source Data. 

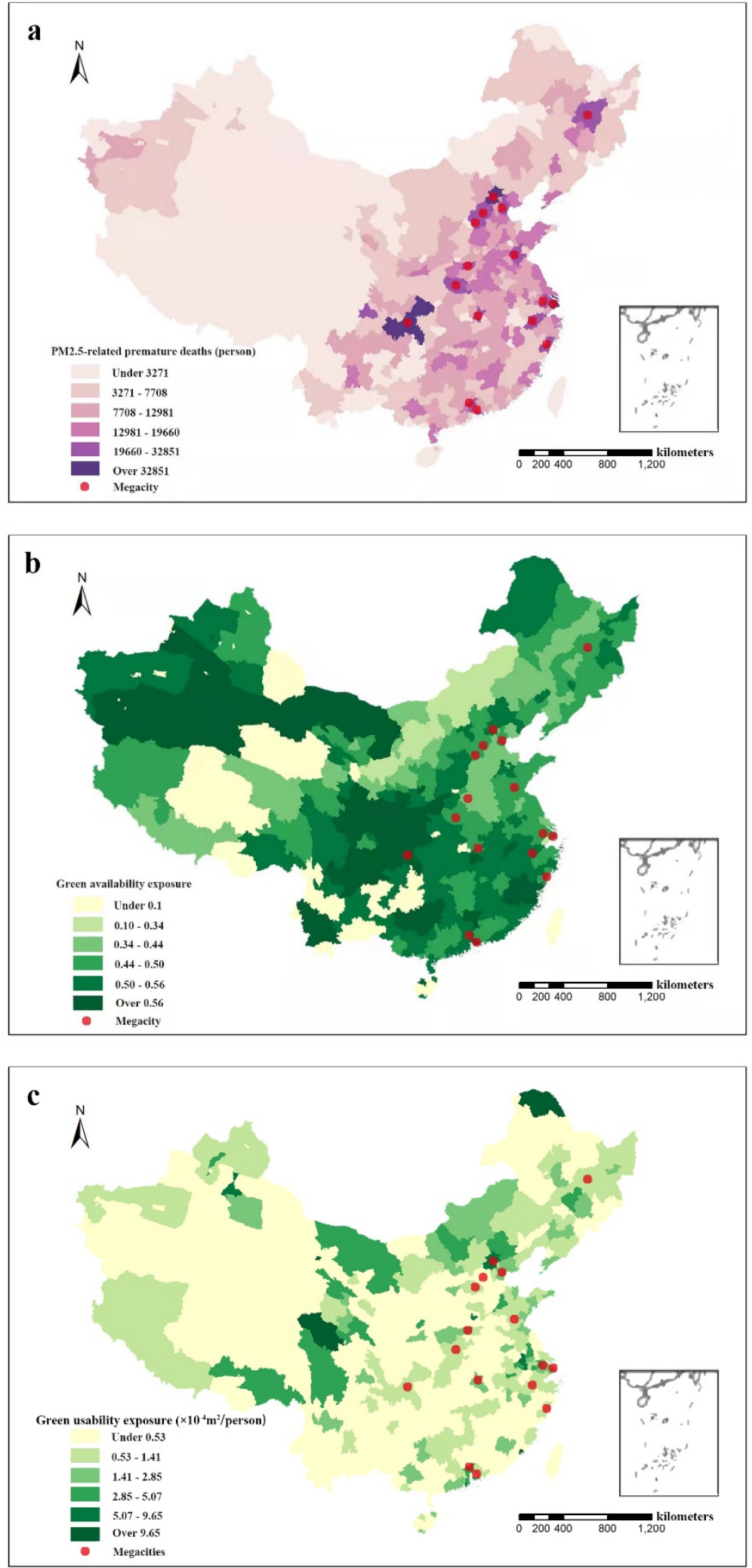

Fig.1 Outdoor $\mathbf{P M}_{2.5}$-related premature deaths (person), Greenspace availability exposure (dimensionless) and Greenspace usability exposure (square meter per person) in 360 China cities. The colors represent different ranges of a annual premature deaths in 2017 , b annual greenspace availability exposure (GAVE) in 2017 and $\mathbf{c}$ annual greenspace usability exposure (GUE) in 2017. 
Overall correlation differences. Table 1 presented the estimated attributable deaths due to outdoor near-ground $\mathrm{PM}_{2.5}$ concentrations by China's cities with demographic characteristic in 2017. The overall multiple linear regression demonstrated that greenspace usability exposure was negatively associated with $\mathrm{PM}_{2.5}$-realted premature deaths, $-0.195(\mathrm{p}<0.001)$ while $\mathrm{PM}_{2.5}$ concentration, population density and GDP had significant positive correlation with them, $0.150,0.116,0.720$ (p $<0.001)$. In the regression analysis, we found that there existed certain outliers which were megacities with a population of more than 10 million. Thus we separated these megacities and compared them with the result of most cities (Supplementary Table 1). We observed that greenspace usability exposure had excellent health benefits generally speaking while the positive effect of greenspace availability exposure on air-pollution health impact was not obvious. For most China cities, greenspace usability as the only negative impact factor $-0.194(\mathrm{p}<0.001)$ could significantly reduce the number of $\mathrm{PM}_{2.5}$-related premature mortalities. $\mathrm{PM}_{2.5}$ concentrations, population density and GDP had positive correlation with air-pollution premature deaths, $0.145,0.112$ and 0.441 ( $\mathrm{p}<$ $0.001)$. In case diagnosis, greenspace had little effect on premature mortality in 17 megacities and GDP as the only significant factor amplified the negative effects of $\mathrm{PM}_{2.5}$ on health 0.619 (p < 0.001 ). This phenomenon had also been reported that capital cities usually showed a high health burden, but greenspace was not sufficient to prevent deaths for the high variability of green levels and interactive influence of multiple factors among capital cities ${ }^{15}$.

\begin{tabular}{|c|c|c|c|c|}
\hline Cities & & All $(n=360)$ & Megacities (n=17) & Other cities $(n=343)$ \\
\hline \multirow{5}{*}{ Factors } & Greenspace availability & 0.047 & 0.850 & 0.015 \\
\hline & Greenspace usability & $-0.195 * * *$ & -0.404 & $-0.194 * * *$ \\
\hline & $\mathbf{P M}_{2.5}$ concentrations & $0.150 * * *$ & 0.065 & $0.145 * * *$ \\
\hline & Population Density & $0.116^{* * *}$ & 0.266 & $0.112 * * *$ \\
\hline & GDP & $0.720 * * *$ & $0.619 * *$ & $0.441 * * *$ \\
\hline $\mathbf{R}^{2}$ & & 0.635 & 0.709 & 0.509 \\
\hline
\end{tabular}

Changes of correlations in urban-rural groups. Table 2, Supplementary Figure 2 showed the changes of greenspace role on estimated attributable deaths due to outdoor near-ground $\mathrm{PM}_{2.5}$ concentrations by urban and rural in Chinese cities, which were divided into urban and rural regions by region code respectively. Because of the existence of megacity cases, we still analyzed them separately. For most cities in China, we observed that greenspace casted a positive impact on mitigating the negative health effects of $\mathrm{PM}_{2.5}$, whether in urban or rural areas. Greenspace usability exposure had a greater negative impact on premature deaths than greenspace availability (Table 2). As the central area transitioned to the countryside, there existed a shift from single mitigation effect of greenspace usability $[-0.098(\mathrm{p}<0.05)]$ to a dual mitigation effect of greenspace availability and greenspace usability [-0.184 $(\mathrm{p}<0.05),-0.280(\mathrm{p}<0.01)]$. Remarkably, the role of GDP in promoting premature deaths weakened $[0.344(\mathrm{p}<0.001)]$ and the positive role of $\mathrm{PM}_{2.5}$ concentrations $[-0.160(\mathrm{p}<0.001)]$ began to increase with the deceleration of urbanization in rural regions. These shifts might be attributed to the sufficient greenspace for small permanent 
population and inadequate promotion of clean energy in rural areas ${ }^{7,26}$. For megacities, the significantly positive effect of GDP on $\mathrm{PM}_{2.5}$ premature deaths was shown in urban regions, 0.595 $(\mathrm{p}<0.001)$ while greenspace usability turned to be clearly negatively correlated with premature deaths in rural regions, $-7.960(\mathrm{p}<0.05)$. This strong disparity could be explained by the highlydeveloped greenspace system and protected forest land in the suburbs to limit urban expansion . In general, greenspace exposure had a greater effect on reducing premature deaths in rural areas than urban areas, and greenspace usability played a better role than greenspace availability. The attributable numbers of greenspace exposure and $\mathrm{PM}_{2.5}$-related deaths by urban and rural, and the differences in other impact factors in 2017 were shown in Source Data.

\begin{tabular}{|c|c|c|c|c|c|}
\hline \multirow{2}{*}{\multicolumn{2}{|c|}{ Cities groups a }} & \multicolumn{2}{|c|}{ Megacities (n=17) } & \multicolumn{2}{|c|}{ Most cities ( $n=343$ ) } \\
\hline & & \multirow{2}{*}{$\frac{\text { Urban }}{0.983}$} & \multirow{2}{*}{$\frac{\text { Rural }}{1.491}$} & \multirow{2}{*}{$\begin{array}{l}\text { Urban } \\
0.023\end{array}$} & \multirow{2}{*}{$\frac{\text { Rural }}{-0.184^{*}}$} \\
\hline Factors & Greenspace availability & & & & \\
\hline & Greenspace usability & 0.806 & $-7.960 *$ & $-0.098 *$ & $-0.280 * *$ \\
\hline & $\mathbf{P M}_{2.5}$ concentrations & -0.029 & 0.438 & $0.059 * * *$ & $0.160 * * *$ \\
\hline & Population Density & -0.032 & 351.034 & 0.017 & -0.024 \\
\hline & GDP & $0.595 * * *$ & 0.011 & $0.415 * * *$ & $0.344 * * *$ \\
\hline \multicolumn{2}{|l|}{$\mathbf{R}^{2}$} & 0.786 & 0.478 & 0.343 & 0.154 \\
\hline
\end{tabular}

Deprivation Index on $\mathbf{P M}_{2.5}$-related premature deaths. Fig. 2 showed the inequity in greenspace exposure and $\mathrm{PM}_{2.5}$-related health burden in 360 cities and four Deprivation Index groups scale. We clustered 360 cities in China based on the Deprivation Index (DI) and divided them into four groups from high to low (Supplementary Figure 3). The higher the DI, the poorer the areas. In general, unequal distribution of greenspace usability in the four DI groups was more obvious than that of greenspace availability, and this inequity corresponded to the difference of $\mathrm{PM}_{2.5}$-related premature deaths in DI groups. Across all urban regions, greenspace availability exposure was highest in highly deprived group and greenspace usability exposure decreased with increasing urban affluence. Across all rural regions, greenspace availability exposure was also highest in 'High' group, greenspace usability exposure was extremely uneven and rising with increasing urban affluence. Through observation we could find that greenspace usability exposure and premature death showed a clear opposite trend, and more inequity was reflected in green usability among DI groups. This results indicated that it was imminent to increase the equivalent opportunities of using greenspace for different poverty level. The clustering subdivision based on Deprivation Index were provided in Supplementary Table $2 \& 3$. 


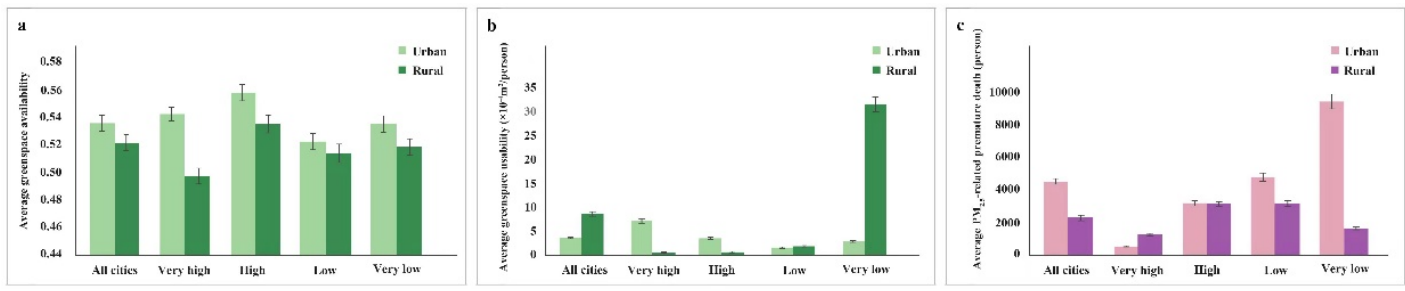

Fig.2 Greenspace exposures and $\mathbf{P M}_{2.5}$-related premature deaths were inequitable in different deprived areas of China. The average greenspace exposure and average $\mathrm{PM}_{2.5}$-related health burden of 360 cities and four Deprivation Index groups were presented with 95\% confidence interval. a average greenspace availability exposure for all cites and four DI groups, $\mathbf{b}$ average greenspace usability exposure for all cites and four DI groups and $\mathbf{c}$ average $\mathrm{PM}_{2.5}$-related premature deaths for all cites and four DI groups.

Table 3, Table 4 and Figure. 3 showed the different relationship of greenspace exposure, other factors and $\mathrm{PM}_{2.5}$-related premature deaths due to Deprivation Index (DI) in urban or rural regions. In general, the common features of urban and rural regions were that greenspace had a significant effect on mitigating $\mathrm{PM}_{2.5}$-related premature deaths in Low and Very low deprived areas. Unlike greenspace exposure, positive effect of GDP and $\mathrm{PM}_{2.5}$ positive weakened as the deprivation level decreased. For urban regions, the richer the area, the more obvious the positive effect of greenspace usability exposure on residents' health, $-0.612(\mathrm{p}<0.001)$ and $-1.212(\mathrm{p}<0.01)$. However, greenspace availability hardly contributed to the health of urban residents related to air pollution (Table 3, Fig. 3). This phenomenon could be explained by that although the urban greenspace was abundant, large amount of greenspace was distributed in the periphery of cities ${ }^{1}$. Meanwhile, high density of urban center construction and uneven distribution of greenspace resources greatly reduced the chances of people being exposed to green environment (Fig. 2), which ultimately led to only increasing greenspace availability was not enough to improve respiratory health ${ }^{25,31}$. A more scientific and effective path was to increase the exposure of green space. Population density had an increasing positive effect of premature deaths in rich area, because high-density human activities would lead to resource shortages and large amount of pollutants emission ${ }^{9}$. High GDP were related to more premature deaths in very highly and highly deprived cities, and $\mathrm{PM}_{2.5}$ concentrations positively influence premature deaths in medium deprived cities. This phenomenon might be explained by the toughest-ever clean air actions in China between 2012-2017, when the combined contributions of strict energy-climate and policy-mandated adjustment were carried out for economic structure ${ }^{32}$. For rural regions, we observed that the positive effect of greenspace availability on $\mathrm{PM}_{2.5}$-related health crisis was on the rise with the increase of affluence. However, greenspace usability showed the opposite trend, and it had no obvious relationship with premature deaths in Very low rural cities. Different from the above results, in the wealthiest rural cities, greenspace availability was the most influential factor for premature deaths and the positive effect on residents' health was much greater than the greenspace availability $-0.397(\mathrm{p}<0.05)$ (Table 4 , Fig. 3). This phenomenon could be comparable to another studies, which found that a 1-unit addition in greenspace availability would led to a certain reduction of potential life, especially in least and greater deprived areas ${ }^{1}$. In addition, an increase in the relationship between $\mathrm{PM}_{2.5}$ concentrations and the promotion of premature death was also observed. This phenomenon had been reported on other researches, which could be attributed to solid fuel consume and resource-intensive 
consumption patterns for rich families ${ }^{7,33,34}$. The distribution of greenspace exposure and $\mathrm{PM}_{2.5^{-}}$ related deaths by DI groups were provided in Source Data.

\begin{tabular}{|c|c|c|c|c|c|}
\hline \multicolumn{6}{|c|}{$\begin{array}{l}\text { Table } 3 \text { Multiple linear relationship between } \mathrm{PM}_{2.5} \text { related premature deaths and } \mathbf{n} \\
\text { urban regions of } 360 \text { cities based on four subdivision groups of deprivation index. } \\
\text { Urban regions }\end{array}$} \\
\hline \multicolumn{2}{|c|}{ Cities groups ${ }^{\mathbf{b}}$} & \multirow{2}{*}{$\begin{array}{l}\text { Very high } \\
(\mathbf{n}=\mathbf{1 3}) \\
-0.012\end{array}$} & \multirow{2}{*}{$\begin{array}{l}\text { High } \\
(\mathbf{n}=\mathbf{1 0 8 )} \\
-0.058\end{array}$} & \multirow{2}{*}{$\begin{array}{l}\text { Low } \\
(\mathbf{n}=176) \\
0.073\end{array}$} & \multirow{2}{*}{$\begin{array}{l}\begin{array}{l}\text { Very low } \\
(\mathbf{n}=\mathbf{6 3})\end{array} \\
0.333\end{array}$} \\
\hline Factors & Greenspace availability & & & & \\
\hline & Greenspace usability & -0.014 & -0.058 & $-0.612^{*}$ & $-1.212^{*}$ \\
\hline & $\mathbf{P M}_{2.5}$ concentrations & 0.011 & $0.073^{*}$ & $0.056^{*}$ & 0.110 \\
\hline & Population Density & 0.041 & $0.186^{*}$ & $-0.089 * *$ & $0.224 * * *$ \\
\hline & GDP & $1.289 * * *$ & 0.205 & $0.608 * * *$ & $0.696 * * *$ \\
\hline \multicolumn{2}{|l|}{$\mathbf{R}^{2}$} & 0.987 & 0.234 & 0.444 & 0.837 \\
\hline
\end{tabular}

\begin{tabular}{|c|c|c|c|c|c|}
\hline & & Urban regi & f 360 cities & & \\
\hline Cities gr & ups $^{b}$ & Very high & High & Low & Very low \\
\hline Factors & Greenspace availability & -0.105 & -0.129 & $-0.251^{*}$ & $-0.397 *$ \\
\hline & Greenspace usability & -7.145 & -0.673 & $-3.836^{* *}$ & -0.135 \\
\hline & $\mathbf{P M}_{2.5}$ concentrations & -0.003 & 0.090 & $0.155^{*}$ & 0.000 \\
\hline & Population Density & -32.447 & 14.092 & $21.726^{*}$ & -0.062 \\
\hline & GDP & $6.092 * * *$ & $3.372 * * *$ & $0.707 * * *$ & 0.025 \\
\hline $\mathbf{R}^{2}$ & & 0.963 & 0.468 & 0.301 & 0.165 \\
\hline
\end{tabular}




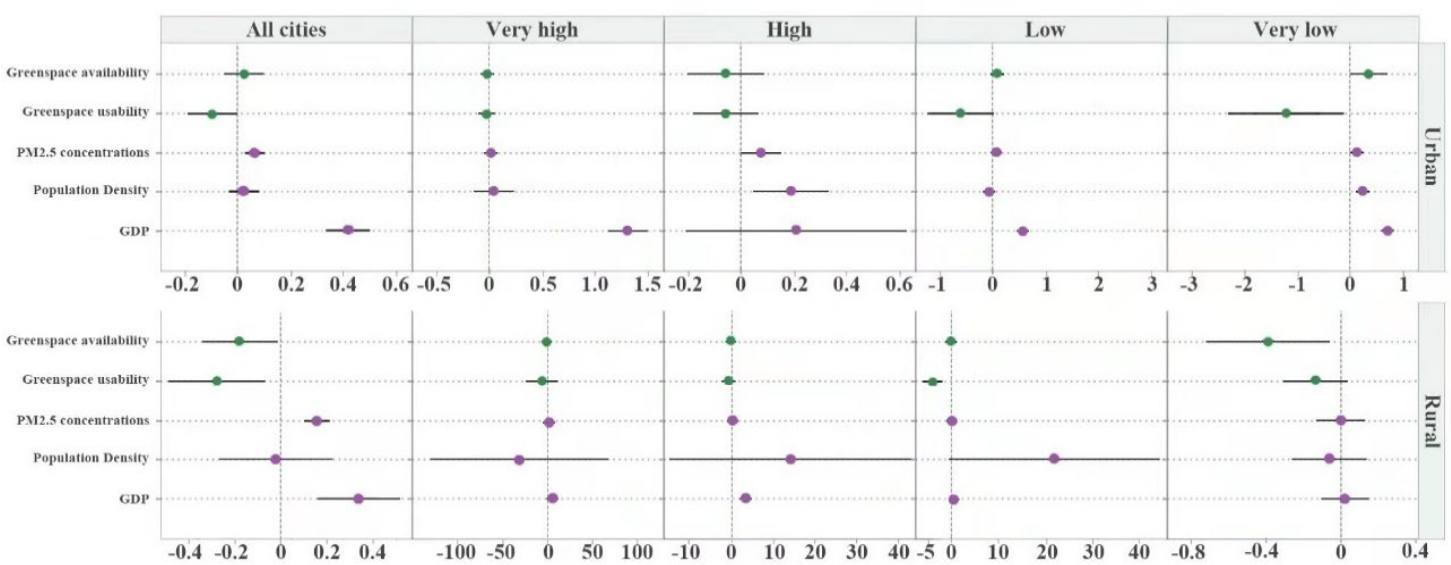

Fig.3 Negative impact of greenspace exposure on $\mathbf{P M}_{2.5}$-related premature deaths showed urban-rural and DI differences. Coefficient values from multiple linear model for the relationship between $\mathrm{PM}_{2.5}$-related premature deaths and greenspace availability exposure (GAVE), greenspace usability exposure (GUE), $\mathrm{PM}_{2.5}$ concentrations, population density and GDP. Coefficient values were represented as dots, bars represent $95 \% \mathrm{Cls}$, greenspace exposures were shown in green and other variables were shown in purple.

\section{Methods}

Integrated modeling framework. Our work has combined multiple data and models from different sources to quantified the impacts of green availability exposure and green usability exposure on $\mathrm{PM}_{2.5}$-related premature deaths in 360 cities of China, and grouped cities according to Deprivation Index for detailed analysis, as listed in Supplementary Table 3. The datasets used in this study include Normalized Difference Vegetation Index (NDVI) from $500 \mathrm{~m}$ resolution MODND1T tenday synthetic products, Leaf Area Index (LAI) from $1 \mathrm{~km}$ resolution MYD15A2 eight-day synthetic products, Land Use-Land Cover (LULC) data from LANDSAT 8 remote sensing image, the dynamic population data in 2017 of China from LandScan (https://landscan.ornl.gov/), the groundlevel $\mathrm{PM}_{2.5}$ mass concentrations data in 2017 from previous study ${ }^{35,36}$, the parameters of IRE model are from the study of Zhao ${ }^{37}$, statistics demographic data in 2017 from the World Population website, the GDP, Per capita disposable income, employment rate, housing prices data are from the National Bureau of Statistics in 2015, the illiteracy rate and the number of education years per person in each city from the data of the $6^{\text {th }}$ National Population Census.

Greenspace availability exposure and Greenspace usability exposure. The greenspace availability exposure index (GAVI) can be expressed by Normalized Difference Vegetation Index (NDVI), Leaf Area Index (LAI) and Land Use-Land Cover (LULC) ${ }^{38}$. Previous studies have proved that NDVI could represents the greenspace density, LAI could measures greenspace quantity, and LULC accounts for the overall presence or absence of greenspace ${ }^{39,40}$. NDVI was obtained from $500 \mathrm{~m}$ resolution, LAI and LULC were obtained with a spatial resolution of $1 \mathrm{~km}$, compared to other moderate resolution, the data were selected due to better higher accuracy. The LULC data contained six land cover types (arable land; woodland; grassland; water; urban-rural, industrial, mining and residential land; unused land), for each land cover type, woodland and grassland was assigned with a value of 1 , other types of land were assigned with a value of 0 , and for each metric, we calculate the proportion of grids with a value of 1 . The previous research interprets that the three indicators had the same weights for contribution of greenspace availability exposure, thus we used the min- 
max normalization method to process these three indicators

At last, we took the three maps to build our availability exposure index through Eq. (1).

$\mathrm{GAVE}_{i}=\frac{\left(G_{N D V I i}+G_{L A I i}+G_{L U L C i}\right)}{P}$

In Eq. (1), GAVE $E_{i}$ is the green availability exposure index value for cell $i$, and $G_{\mathrm{NDVli}}, \mathrm{G}_{\mathrm{LAIi}}$ and $G_{\text {LULCi }}$ are greenspace metric 'exposure' values for corresponding cell i. $P$ is the number of metrics (in our case, $\mathrm{P}=3$ ). In this study, $\mathrm{GAVE}_{\mathrm{i}}$ values ranged between 0 and 1 , in which 1 indicates the highest availability exposure of greenspace, and 0 means the lowest level, or no available of greenspace exposure. Remarkably, we removed and set the negative NDVI values to zero in our computation, as these values expressed water or other non-green covers. We resampled the GAVE map to $1 \mathrm{~km}$ resolution using ArcGIS (v 10.2) to be consistent with other exposure layers.

The greenspace usability exposure (GUE) is described by dynamic population and land use data in 2017 , China ${ }^{17}$. Since the individual location is temporally varied, the model to objectively reflect people's real-time use of greenspace is established for evaluating the greenspace usability exposure. Thereby presenting a more reasonable way to access population exposure to greenspace ${ }^{20}$. By integrating the dynamic population distribution and land use data into the assessment with a spatial resolution of $1 \mathrm{~km}$, we further modified the model in Eq. (2) in a dynamic population-weight manner. The green usability exposure $\left(\mathrm{GUE}_{\mathrm{i}}\right)$ is estimated as Eq. (2).

$\mathrm{GUE}_{i}=\frac{\sum_{i=1}^{n} S i}{\sum_{i=1}^{n} P i}$

In Eq. (2), $S i$ represents the green area of cell i, $P i$ is the dynamic population within cell i. GUE is greenspace metric 'usability exposure' values for corresponding cell i. In this study, $\mathrm{GUE}_{\mathrm{i}}$ values also ranged between 0 and 1 , in which 1 indicates the highest usability exposure of greenspace, and 0 means the lowest level.

Estimating $\mathbf{P M}_{2.5}$-related premature deaths. We use satellite-based ground-level $\mathbf{P M}_{2.5}$ concentrations data and the IER model to estimate $\mathrm{PM}_{2.5}$-related premature deaths. The IER model is developed by Burnett, it describes the concentration-response relationship for the overall range of $\mathrm{PM}_{2.5}$ concentration observed in the world ${ }^{41}$. And it also has been used in many influential studies to estimate the $\mathrm{PM}_{2.5}$-related premature deaths ${ }^{42,43,44}$. In this study, we use IER model to estimate the total number by four main causes of the $\mathrm{PM}_{2.5}$-related premature deaths: ischemic heart disease (IHD), stroke, chronic obstructive pulmonary disease (COPD), and lung cancer (LC). The relative risk (RR) for each disease is calculated as Eq. (3).

$\operatorname{RR}\left(C_{P M 2.5}\right)=\left\{\begin{array}{cl}1+a_{1}\left(1-e^{-a_{2}\left(C_{P M 2.5}-C_{0}\right)^{a_{3}}}\right), & \text { if } C_{P M 2.5}>C_{0} \\ 1, & \text { else }\end{array}\right.$

where $\mathrm{C}_{\mathrm{PM} 2.5}$ is the satellite-based $\mathrm{PM}_{2.5}$ concentrations in 2017; $\mathrm{C}_{0}$ is the counterfactual concentration, in this study, there is assumed to be no additional risk below $\mathrm{C}_{0} ; \mathrm{a}_{1}, \mathrm{a}_{2}$ and $\mathrm{a}_{3}$ are parameters to describe the overall shape of the concentration response, the parameters which we used in this study are adopted from Lee et al. ${ }^{45}$, and the values are listed in Supplementary Table 4. The deaths due to $\mathrm{PM}_{2.5}$ pollution are calculated as Eq. (4).

$M_{P M 2.5}=\frac{\operatorname{RR}\left(C_{P M 2.5}\right)-1}{\operatorname{RR}\left(C_{P M 2.5}\right)} \times B_{C} \times P_{C}$

where $\mathrm{M}_{\mathrm{PM} 2.5}$ is the total deaths related to $\mathrm{PM}_{2.5} ; \frac{R R-1}{R R}$ is the attributable proportion to $\mathrm{PM}_{2.5}$ pollution; derived from the national average data in GBD $2013^{46}, B_{C}$ is the baseline incidence for 
all age group of a given health endpoint; $P_{C}$ is the scale of the exposed population gathered from the Word Population database in 2017 at a $1 \mathrm{~km}$ resolution. Value for $B_{C}$ used in this study are in Supplementary Table 4.

Linking premature deaths to different influencing factors. In this study, multiple linear regression is used to identify the relationship between influencing factors $\mathrm{PM}_{2.5}$-related premature deaths: green availability exposure, green usability exposure, $\mathrm{PM}_{2.5}$ concentrations, population density and GDP. The $\mathrm{PM}_{2.5}$ concentrations, GDP and population density maps of 360 China cities in 2017 are presented in Fig. 1. PM $_{2.5}$ concentrations has been proved to be strongly associated with premature death ${ }^{47}$. Except for greenspace exposure, previous studies have shown that $\mathrm{PM}_{2.5}$ concentrations, population density and GDP also had a strong correlation with air pollution-related health impact ${ }^{48}$.

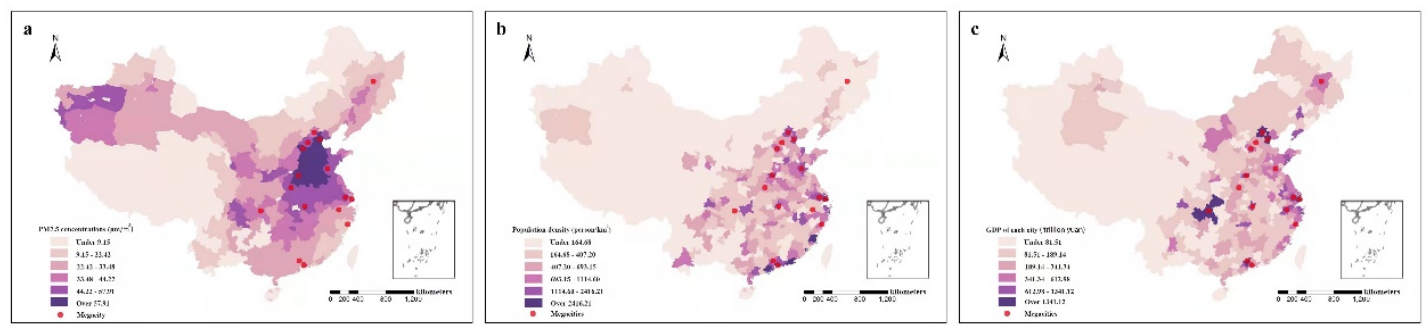

Fig.4 PM $_{2.5}$ concentrations (microgram per cubic meter), Population density (person per square kilometer) and GDP (100 million yuan) in $\mathbf{3 6 0}$ China cities. The colors represent different ranges of a annual mean $\mathrm{PM}_{2.5}$ concentrations in 2017, $\mathbf{b}$ annual population density in 2017 and $\mathbf{c}$ annual Gross Domestic Product in 2015.

The regression coefficients derived from the MLR analysis can fully reflect the sensitivity of the dependent variable $\left(\mathrm{PM}_{2.5}\right.$-related premature deaths) to multiple independent variables (green availability exposure, green usability exposure, $\mathrm{PM}_{2.5}$ concentrations, GDP and population density) 49. The MLR models were constructed as Eq. (4).

$\mathrm{M}_{\mathrm{PM} 2.5}=\gamma_{1} \times G A V E_{i}+\gamma_{2} \times G U E_{i}+\gamma_{3} \times C_{P M 2.5}+\gamma_{4} \times P D+\gamma_{5} \times G D P$

where $G A V E_{i}$ is the greenspace availability exposure; $G U E_{i}$ is the greenspace usability exposure; $\mathrm{C}_{\mathrm{PM} 2.5}$ is the $\mathrm{PM}_{2.5}$ concentrations; PD represent the population density; GDP represent the Gross Domestic Product; $\gamma_{1}, \gamma_{2}, \gamma_{3}, \gamma_{4}, \gamma_{5}$ are the regression coefficient values represent the influence degree of the independent variable. The regression coefficient represents the positive and negative correlation between dependent variables and independent variables, and the magnitude of the coefficient indicates the strength of the correlation.

Subdivided groups. Previous study provided the correlation of $\mathrm{PM}_{2.5}$ with mortality risks and illustrated urban-rural difference of $\mathrm{PM}_{2.5}$-related premature death ${ }^{50}$. In this study, we use the 'Zoning and Urban and Rural Codes' of the National Bureau of Statistics for 2017 to divide urban and rural areas among 360 cities of China to discuss the disparities $\mathrm{PM}_{2.5}$-related premature death between urban and rural areas under different dependent variables. The code is widely used in various statistical surveys and urban and rural management, which is of great significance to multiple statistical works.

The standard of deprivation level has changed from single dimension to multi-dimension in recent years, the identification dimensions and methods of deprivation level have been relatively improved, and the method Alkire and Foster have proposed is widely used to measure deprivation level of a certain area from the aspects of income, education, health and other aspects ${ }^{51}$. Based on many 
relative studies ${ }^{52,53,54}$, we choose the following four aspects as the measures of deprivation index, income level, educational level, employment level and living standard. Limited to data acquisition, we select one or two indicators for each aspect, we use urban and rural per capita disposable income, illiteracy rate, average years of education, urban unemployment rate and commodity housing price to estimate the deprivation level, and different deprivation indexes are obtained through min-max. Through K-means cluster analyze, we divided the deprivation index into four groups, very high, high, low and very low, the result of k-means is listed in Supplementary Table 2.

\section{Discussion}

This work developed the correlation between greenspace exposure and $\mathrm{PM}_{2.5}$-related premature death for the first time, and the per capital greenness was firstly used to characterize greenspace usability exposure. Using the urban-rural code and cluster analysis method, we divided 360 China's cities into urban-rural and deprivation level group. Although substantial contribution

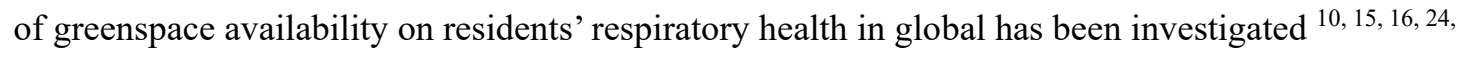
55 , we found that the positive impact of greenspace usability exposure on premature death was significantly stronger than that of greenspace availability exposure in most situations, while greenspace availability exposure only worked in the more affluent rural areas. These findings further emphasize the great importance of increasing per capital greenspace exposure, given that current policies focus more on greenspace equality instead of paying much attention to the greenspace availability exposure ${ }^{1,26,56}$. Additionally, $\mathrm{PM}_{2.5}$ concentrations, population density and GDP promoted $\mathrm{PM}_{2.5}$-related premature deaths as expected, but the positive effect of $\mathrm{PM}_{2.5}$ concentrations was greatly weakened compared with other studies ${ }^{8,32}$. From 2013 to 2017, the Chinese government was committed to air pollution control, but the latest research showed that a sharp drop in $\mathrm{PM}_{2.5}$ will lead to the rise of other types of pollutants, which would increase health burdens ${ }^{57}$. This reminds us that from the perspective of promoting residents' health, policies of controlling pollution source were important, but improving city ecological environment to increase slow-release pathways for emissions was much more important and sustainable.

Our work provides unprecedented insight into the different positive effects of greenspace availability and usability exposure on $\mathrm{PM}_{2.5}$-related premature death with urban-rural changes. Moreover, we highlight systematic differences in the impacts of greenspace exposure on $\mathrm{PM}_{2.5-}$ related premature deaths according to city deprivation level and location. For example, we show that greenspace usability exposure in richest urban regions affects mortalities most while greenspace availability exposure in richest rural regions affect mortalities most. These findings point to targeted opportunities for greenspace increment, such as construct informal green spaces to increase the per capita green space exposure of urban residents and increase the green patch area to alleviate the air pollution-related health impact of rural residents ${ }^{15}$. But more importantly, our work offers a basis for greenspace planning policies that avoid and redress socioeconomic regional inequities. For urban regions, high concentration of urbanization makes it difficult to build large areas of greenspace, and the total amount of greenspace is no longer a major factor in improving the living environment of residents. We suggest that focus on improving the community proximity and quality of urban green space, and building roof gardens or other public open greenspace in the affluent urban regions to relieve the high-density population and increase the opportunities of greenspace exposure ${ }^{56}$. Such as Beijing inserting greenspace policy and the UK's 300-meter accessible natural green space plan

${ }^{58}$. For rural regions, the direct emissions from solid fuels burned by rural residents and the lack of 
greenspace availability in residential clusters remind that we should increase the equity of rural residents' greenspace exposure and vigorously promote the use of clean energy ${ }^{7,25,59}$. For example, in Kondo of USA, increasing the tree canopy cover made the poorer rural neighborhoods benefit more ${ }^{15,58}$. Since these changes must be borne by highly-deprived regions, consumption-based policies can better support the required technology transfer and capital investment ${ }^{7,60}$.

Our study is subject to several uncertainties and limitation from the use of multiple datasets and analyzing method. First, the IER model used in mortality estimates are are uncertain due to that the adaptation degree of IER model to $\mathrm{PM}_{2.5}$-related indexes in China is not clear yet ${ }^{61}$. Using concentration-response relationships based on local cohort studies would improve the accuracy in estimating of premature deaths in the future ${ }^{7}$. Second, deprivation level assessment is not comprehensive enough due to the lack of complete data of residential and crime rate statistics. Improvement of statistical data collection system or conducting field surveys could remedy this situation in the future. Third, multiple linear regression contributes to the uncertainties in estimating the relationship between greenspace exposure and $\mathrm{PM}_{2.5}$ related mortalities for each group. Explaining the cross-correlation among latent variables by structural equation model could improve the identification of correlation of greenspace exposure and premature mortalities in the future ${ }^{62}$. 


\section{References}

1. Labib SM, Lindley S, Huck JJ. Estimating multiple greenspace exposure types and their associations with neighbourhood premature mortality: A socioecological study. The Science of the total environment 789, 147919 (2021).

2. Maji KJ, Dikshit AK, Arora M, Deshpande A. Estimating premature mortality attributable to PM2.5 exposure and benefit of air pollution control policies in China for 2020. The Science of the total environment 612, 683-693 (2018).

3. Lelieveld J, Evans JS, Fnais M, Giannadaki D, Pozzer A. The contribution of outdoor air pollution sources to premature mortality on a global scale. Nature 525, 367-371 (2015).

4. Zhang Q, et al. Transboundary health impacts of transported global air pollution and international trade. Nature 543, 705-709 (2017).

5. Chowdhury S, Dey S, R. Smith K. Ambient PM2.5exposure and expected premature mortality to 2100 in India under climate change scenarios. Nature communications $\mathbf{9}, 318$ (2018).

6. McDuffie EE, et al. Source sector and fuel contributions to ambient PM2.5 and attributable mortality across multiple spatial scales. Nature communications 12, 3594 (2021).

7. Zhao $\mathrm{H}$, et al. Inequality of household consumption and air pollution-related deaths in China. Nature communications 10, 4337 (2019).

8. Lim CH, Ryu J, Choi Y, Jeon SW, Lee WK. Understanding global PM2.5 concentrations and their drivers in recent decades (1998-2016). Environment internationa/144, 106011 (2020).

9. Sun $\mathrm{S}$, et al. Air pollution associated respiratory mortality risk alleviated by residential greenness in the Chinese Elderly Health Service Cohort. Environmental research 183, 109139 (2020).

10. Sun $\mathrm{Y}$, et al. Associations between green space and preterm birth: Windows of susceptibility and interaction with air pollution. Environment international 142, 105804 (2020).

11. Ferrante G, Asta F, Cilluffo G, De Sario M, Michelozzi P, La Grutta S. The effect of residential urban greenness on allergic respiratory diseases in youth: A narrative review. The World Allergy Organization journa/ 13, 100096 (2020).

12. Wu J, Yang M, Xiong L, Wang C, Ta N. Health-oriented vegetation community design: Innovation in urban green space to support respiratory health. Landscape and Urban Planning 205, 103973 (2021). 
13. Su F, et al. Rapid greening response of China\&\#x2019;s 2020 spring vegetation to COVID19 restrictions: Implications for climate change. Science Advances 7, eabe8044 (2021).

14. Nieuwenhuijsen $\mathrm{M}$, Khreis $\mathrm{H}$. Green space is important for health. The Lancet $\mathbf{3 8 9}, 700$ (2017).

15. Barboza EP, et al. Green space and mortality in European cities: a health impact assessment study. The Lancet Planetary Health 5, e718-e730 (2021)

16. Ge E, et al. Greenness exposure and all-cause mortality during multi-drug resistant tuberculosis treatment: A population-based cohort study. The Science of the total environment 771, 145422 (2021).

17. Song $\mathrm{Y}$, Chen B, Kwan M-P. How does urban expansion impact people's exposure to green environments? A comparative study of 290 Chinese cities. Journal of Cleaner Production 246, 119018 (2020).

18. Triguero-Mas M, et al. Natural outdoor environments and mental and physical health: relationships and mechanisms. Environment internationa/ 77, 35-41 (2015).

19. de Keijzer C, et al. The association of air pollution and greenness with mortality and life expectancy in Spain: A small-area study. Environment internationa/99, 170-176 (2017).

20. Song YM, Huang B, Cai JX, Chen B. Dynamic assessments of population exposure to urban greenspace using multi-source big data. Science of the Total Environment 634, 13151325 (2018).

21. Mitchell R, Popham F. Effect of exposure to natural environment on health inequalities: an observational population study. The Lancet 372, 1655-1660 (2008).

22. Spotswood EN, et al. Nature inequity and higher COVID-19 case rates in less-green neighbourhoods in the United States. Nature Sustainability, (2021).

23. Mears M, Brindley $P$, Jorgensen A, Ersoy E, Maheswaran R. Greenspace spatial characteristics and human health in an urban environment: An epidemiological study using landscape metrics in Sheffield, UK. Ecological Indicators 106, 105464 (2019).

24. Lachowycz K, Jones AP. Does walking explain associations between access to greenspace and lower mortality? Social science \& medicine 107, 9-17 (2014).

25. Tian M, Yuan L, Guo R, Wu Y, Liu X. Sustainable development: Investigating the correlations between park equality and mortality by multilevel model in Shenzhen, China. Sustainable Cities and Society 75, 103385 (2021). 
26. Kasdagli MI, Katsouyanni K, de Hoogh K, Lagiou P, Samoli E. Associations of air pollution and greenness with mortality in Greece: An ecological study. Environmental research 196, 110348 (2021).

27. Klompmaker JO, et al. County-level exposures to greenness and associations with COVID19 incidence and mortality in the United States. Environmental research 199, 111331 (2021).

28. Pearsall $\mathrm{H}$, et al. Advancing equitable health and well-being across urban-rural sustainable infrastructure systems. npj Urban Sustainability 1 , (2021).

29. Rao ND, Kiesewetter G, Min J, Pachauri S, Wagner F. Household contributions to and impacts from air pollution in India. Nature Sustainability 4, 859-867 (2021).

30. Gourevitch JD, et al. Projected losses of ecosystem services in the US disproportionately affect non-white and lower-income populations. Nature communications 12, 3511 (2021).

31. Chen $Y$, et al. Inequalities of urban green space area and ecosystem services along urban center-edge gradients. Landscape and Urban Planning 217, 104266 (2022).

32. Geng G, et al. Drivers of PM2.5 air pollution deaths in China 2002-2017. Nature Geoscience 14, 645-650 (2021).

33. Shen $\mathrm{H}$, et al. Urbanization-induced population migration has reduced ambient PM2.5 concentrations in China. Science Advances 3, e1700300 (2017).

34. Yun X, et al. Residential solid fuel emissions contribute significantly to air pollution and associated health impacts in China. Science Advances 6, eaba7621 (2020).

35. He Q, Gao K, Zhang L, Song Y, Zhang M. Satellite-derived 1-km estimates and long-term trends of PM2.5 concentrations in China from 2000 to 2018. Environment international 156, 106726 (2021).

36. He QQ, Gu YF, Zhang M. Spatiotemporal trends of PM2.5 concentrations in central China from 2003 to 2018 based on MAIAC-derived high-resolution data. Environment internationa/ 137, (2020).

37. Zhao HY, et al. Inequality of household consumption and air pollution-related deaths in China. Nature Communications 10, (2019).

38. Labib SM, Lindley S, Huck JJ. Estimating multiple greenspace exposure types and their associations with neighbourhood premature mortality: A socioecological study. Science of The Total Environment 789, 147919 (2021). 
39. Engemann K, Pedersen CB, Arge L, Tsirogiannis C, Mortensen PB, Svenning JC. Residential green space in childhood is associated with lower risk of psychiatric disorders from adolescence into adulthood. Proceedings of the National Academy of Sciences of the United States of America 116, 5188-5193 (2019).

40. James P, Banay RF, Hart JE, Laden F. A Review of the Health Benefits of Greenness. Current epidemiology reports 2, 131-142 (2015).

41. Burnett RT, et al. An Integrated Risk Function for Estimating the Global Burden of Disease Attributable to Ambient Fine Particulate Matter Exposure. Environmental Health Perspectives 122, 397-403 (2014).

42. Yang XC, et al. Health risk and disease burden attributable to long-term global fine-mode particles. Chemosphere 287, (2022).

43. Maji KJ, Dikshit AK, Arora M, Deshpande A. Estimating premature mortality attributable to PM2.5 exposure and benefit of air pollution control policies in China for 2020. Science of the Total Environment 612, 683-693 (2018).

44. Johnston FH, et al. Unprecedented health costs of smoke-related PM2.5 from the 201920 Australian megafires. Nature Sustainability 4, 42-47 (2021).

45. Lee CJ, Martin RV, Henze DK, Brauer M, Cohen A, van Donkelaar A. Response of Global Particulate-Matter-Related Mortality to Changes in Local Precursor Emissions. Environmental Science \& Technology 49, 4335-4344 (2015).

46. Forouzanfar MH, Afshin A, Alexander LT, al. e. Global, regional, and national comparative risk assessment of 79 behavioural, environmental and occupational, and metabolic risks or clusters of risks, 1990-2015: a systematic analysis for the Global Burden of Disease Study 2015. Lancet 388, 1659-1724 (2016).

47. Apte JS, Marshall JD, Cohen AJ, Brauer M. Addressing Global Mortality from Ambient PM2.5. Environmental Science \& Technology 49, 8057-8066 (2015).

48. Xie Y, Dai HC, Dong HJ, Hanaoka T, Masui T. Economic Impacts from PM2.5 PollutionRelated Health Effects in China: A Provincial-Level Analysis. Environmental Science \& Technology 50, 4836-4843 (2016).

49. He Z, Liu P, Zhao X, He X, Liu J, Mu Y. Responses of surface O3 and PM2.5 trends to changes of anthropogenic emissions in summer over Beijing during 2014-2019: A study based on multiple linear regression and WRF-Chem. Science of The Total Environment 807, 150792 (2022).

50. Liu T, et al. Urban-rural disparity of the short-term association of PM2.5 with mortality 
and the mortality burden attributable to ambient PM2.5 in China. The Innovation, 100171 (2021).

51. Alkire S, Foster J. Counting and multidimensional poverty measurement. Journal of Public Economics 95, 476-487 (2011).

52. Omar MA, Hasanujzaman M. Multidimensional energy poverty in Bangladesh and its effect on health and education: A multilevel analysis based on household survey data. Energy Policy 158, 112579 (2021).

53. Zhang Z, Ma C, Wang A. A longitudinal study of multidimensional poverty in rural China from 2010 to 2018. Economics Letters 204, 109912 (2021).

54. Bárcena-Martín E, Pérez-Moreno S, Rodríguez-Díaz B. Rethinking multidimensional poverty through a multi-criteria analysis. Economic Modelling 91, 313-325 (2020).

55. $\mathrm{Yu} \mathrm{H}$, et al. Association between eye-level greenness and lung function in urban Chinese children. Environmental research 202, 111641 (2021).

56. Mears M, Brindley $P$, Jorgensen A, Ersoy E, Maheswaran R. Greenspace spatial characteristics and human health in an urban environment: An epidemiological study using landscape metrics in Sheffield, UK. Ecological Indicators 106, (2019).

57. Li K, et al. A two-pollutant strategy for improving ozone and particulate air quality in China. Nature Geoscience 12, 906-910 (2019).

58. Barbosa O, et al. Who benefits from access to green space? A case study from Sheffield, UK. Landscape and Urban Planning 83, 187-195 (2007).

59. Wang S, Liu X, Yang X, Zou B, Wang J. Spatial variations of PM2.5 in Chinese cities for the joint impacts of human activities and natural conditions: A global and local regression perspective. Journal of Cleaner Production 203, 143-152 (2018).

60. Ramaswami A, et al. Urban cross-sector actions for carbon mitigation with local health co-benefits in China. Nature Climate Change 7, 736-742 (2017).

61. Chen Z, Wang JN, Ma GX, Zhang YS. China tackles the health effects of air pollution. Lancet 382, 1959-1960 (2013).

62. Murray ET, Nicholas O, Norman P, Jivraj S. Neighbourhood deprivation effects on bodymass index across the life course: structural equation modelling of data from two prospective British longitudinal birth cohorts. The Lancet 394, S90 (2019). 


\section{Supplementary Files}

This is a list of supplementary files associated with this preprint. Click to download.

- SourceData.rar

- supplementary.docx 